\title{
Final Report: Thermal Conductance of Solid-Liquid Interfaces
} US DOE Grant No. DEFG02-01ER45938, Sept. 15, 2001 to Sept. 15, 2005.

\author{
David G. Cahill and Paul V. Braun \\ Frederick Seitz Materials Research Laboratory, University of Illinois at Urbana-Champaign
}

\section{Summary of Main Accomplishments}

Research supported by this grant has significantly advanced fundamental understanding of the thermal conductance of solid-liquid interfaces, and the thermal conductivity of nanofluids and nanoscale composite materials.

- The thermal conductance of interfaces between carbon nanotubes and a surrounding matrix of organic molecules is exceptionally small and this small value of the interface conductance limits the enhancement in thermal conductivity that can be achieved by loading a fluid or a polymer with nanotubes [1].

- The thermal conductance of interfaces between metal nanoparticles coated with hydrophilic surfactants and water is relatively high and surprisingly independent of the details of the chemical structure of the surfactant [2].

- We extended our experimental methods to enable studies of planar interfaces between surfactant-coated metals and water where the chemical functionalization can be varied between strongly hydrophobic and strongly hydrophilic. The thermal conductance of hydrophobic interfaces establishes an upper-limit of $0.25 \mathrm{~nm}$ on the thickness of the vapor-layer that is often proposed to exist at hydrophobic interfaces [3].

- Our high-precision measurements of fluid suspensions show that the thermal conductivity of fluids is not significantly enhanced by loading with a small volume fraction of spherical nanoparticles. These experimental results directly contradict some of the anomalous results in the recent literature and also rule-out proposed mechanisms for the enhanced thermal conductivity of nanofluids that are based on modification of the fluid thermal conductivity by the coupling of fluid motion and the Brownian motion of the nanoparticles [4].

\section{A. Research Accomplishments}

\section{A.1 Nanoparticle suspensions and composites}

Our studies of heat transport at the interfaces between a metal nanoparticle and a surrounding liquid $[5,6]$ were motivated by the excitement generated by reports of anomalously high thermal conductivities of nanoparticle suspensions. Effective medium theories predict an rigorous upper limit to the relative enhancement in the thermal conductivity of a suspension of spherical particles at $3 f$, where $f$ is the volume fraction of particles; i.e., a $1 \mathrm{vol} \%$ percent loading of nanoparticles in a fluid should produce no more than a $3 \%$ increase in the thermal conductivity. In many cases, the effective medium theories of isolated or aggregated particles is in reasonably good agreement with experiment [7]. But in some cases, anomalously 
large enhancements have been observed. None of the anomalous results have been verified independently.

Recently, we reported our measurements of the thermal conductivity of nanoparticle suspensions [4] using a novel micron-scale optical-beam-deflection method operating at $\approx 30 \mathrm{~Hz}$. In contrast to prior reports of anomalously enhanced thermal conductivity, we do not observe a significant increase in thermal conductivity outside the precision of our measurements of $\pm 1 \%$. Our result also contradicts speculations that nanoscale convection created by the Brownian motion of nanoparticles can create large enhancements in the thermal conductivity of dilute suspensions [8].

In a nanoscale composite, the thermal conductance of the interfaces plays a critical role in determining the properties of the composite. For example, in a composite fluid or material comprised of high thermal conductivity spherical particles dispersed in a low thermal conductivity matrix, the particles will enhance the thermal conductivity of the composite only if $r>r_{c}$ where $r_{c}=\Lambda / G, \Lambda$ is the thermal conductivity of the matrix and $G$ is the thermal conductance per unit area of the particle/matrix interface. While $G$ had been measured for many combinations of solids, prior to our work, $G$ for solid-liquid or solid-polymer interfaces was unknown.

To directly measure $G$ and more generally probe the heat transport in the immediate surroundings of a metal nanoparticle, we measured the cooling rates of metal nanoparticles in suspension by time-resolved optical absorption [5, 2]. Surprisingly, we found that the interfaces between metals and water was essentially independent of the structure of the molecular layers used to stabilize the suspension; for Au nanoparticles suspended in water, $r_{c} \approx 3 \mathrm{~nm}[2]$.

\section{A.2 Thermal conductance of hydrophobic and hydrophilic interfaces.}

The structure and thermodynamics of water near the surfaces of solids or macromolecules is an extremely active area of interdisciplinary research. A greater understanding of the watermaterial interface has broad implications for current scientific problems as diverse as the biological activity of proteins and the desalination of water by reverse osmosis membranes.

Our use of transient absorption measurements of nanoparticle suspensions has provided the first quantitative data for the thermal conductance of solid-liquid interfaces but the combination of materials, surfactants, and liquids that we can study using this approach is limited. For example, the thermal conductance of simple hydrophobic interfaces in water cannot be studied in this way because particles with electrically neutral hydrophobic surfaces will rapidly form large aggregates.

To overcome this limitation, we have recently developed an experimental approach for measuring the thermal conductance of solid-liquid interfaces using time-domain thermoreflectance of a planar metal film in contact with water [3]. A $30 \mathrm{~nm}$ thick Al film serves as the heater and thermometer in the experiment. The Al layer can be coated directly by various silicon-organic molecules or coated with thin layers of $\mathrm{Ti} / \mathrm{Au}$ and then coated by alkanethiol chemistries to provide various functionalities at the interface between the molecular monolayer and water. 


\section{A.3 Carbon nanotubes and fullerene molecules}

For a composite material that incorporates high aspect ratio, high thermal conductivity fibers, there is no simple upper limit to the thermal conductivity of the composite. In the limit of high aspect ratio, and ignoring interface effects, the thermal conductivity of an isotropic fiber composite is $\left(v_{f} / 3\right) \Lambda_{f}$, where $v_{f}$ is the volume fraction of fibers and $\Lambda_{f}$ is the thermal conductivity of the fiber. Since carbon nanotubes have been reported to have extremely high thermal conductivities, we might expect that a nanotube composite would have a high thermal conductivity. Many studies have shown that this is not the case. Our work, in collaboration with Pawel Keblisnki at RPI, showed that the small thermal conductance of the interfaces between the nanotubes and the surrounding fluid or polymer greatly limits the thermal conductivity of the composite $[1,9]$.

To quantify the thermal conductance of nanotube-matrix interfaces, we applied transient absorption and studied a model system of nanotubes suspended in water and stabilized by a standard surfactant, SDS [1]. Suspensions of single-walled nanotubes were supplied by a collaboration with Michael Strano's group in the Chemical Engineering Department of UI. The immediate neighborhood of the nanotube is alkane end of the surfactant. The result of our measurements is $G=12 \mathrm{MW} \mathrm{m}^{-2} \mathrm{~K}^{-1}$, which agreed well with computational models.

The interpretation of these experiments are complicated by the fact that the not all of the vibrational modes of the nanotubes are in thermal equilibrium. The computational experiments show that the vast majority of the vibrational modes of the nanotube are at the same temperature but a small fraction of the lowest energy modes are more tightly coupled to the surroundings than to the remainder of the modes in the tube. Therefore, the thermal conductance includes two components that act in series: i) heat transfer between the large number of high frequency modes that dominate the heat capacity into the small number of low frequency modes and ii) heat transfer between the low frequency modes of the tubes and the surrounding fluid. We further tested these ideas with experimental and computational studies of the vibrational energy relaxation of fullerene molecules dissolved in a variety of organic solvents [10].

\section{A.4 Nanoscale polymer layers}

Thermal transport in the immediate vicinity of a nanoparticles can also be probed using transient absorption as long as the dimensionless parameter $r G / \Lambda$ is not too small. In collaboration with Andrew Taton's group at the U. Minnesota, we studied thermal transport in Au core-polymer shell nanoparticles [6]. The polymer shell is made of a co-polymer of polystyrene and polyacrylic acid that provides a hydrophilic outer surface and a hyrophobic inner surface. In this way, the Au core is well protected from water molecules and ions in aqueous solutions. When a co-solvent such as tetrahydrofuran is added to the suspension, the co-polymer shell swells and we discovered a surprising change in the thermal transport properties of the shell; the cooling rate of the particles increases by a factor of 2 at the same composition of co-solvent that initiates swelling of the polymer shell. We are uncertain of the cause of this change in transport properties - the amount of water in the shell is too small to explain the change using simple effective medium theory - but if we attribute the change in cooling rate to a change in the thermal conductivity of the shell, the thermal conductivity 
of the shell increases by a factor of 2 with swelling [6].

\section{B. Support of Graduate Research Assistants and Post-doctoral Research Associates}

The education and training of two graduate research assistants, Zhenbin Ge and Shawn Putnam, and two post-doctoral research associates, Scott Huxtable and Ho-Ki LYeo, was supported by this grant. Dr. Ge recently completed his Ph.D. degree $[2,6,3]$ and is employed by Applied Materials. Shawn Putnam's work on the thermal conductivity of nanoparticle suspensions [4] and the thermodiffusion [11] of metallic nanoparticles was supported by this grant and we expect Mr. Putnam to complete his Ph.D. degree at the end of this summer. Scott Huxtable $[1,12,13,10]$ is now an assistant professor at Virginia Tech; Prof. Huxtable received a CAREER award from the NSF in 2006. Ho-Ki Lyeo's work on the thermal conductance of interfaces between materials with highly dissimilar vibrational spectra [14] was supported by this grant; Dr. Lyeo is now a staff scientist at the Korean Research Institute for Standards and Science.

\section{Publications}

[1] S. T. Huxtable et al., "Interfacial heat flow in carbon nanotube suspensions," Nature Materials 2, 731-734 (2003).

[2] Z. Ge, D. G. Cahill, and P. V. Braun, "AuPd metal nanoparticles as probes of nanoscale thermal transport in aqueous solution," Journal of Physical Chemistry B 108, 18870 18875 (2004).

[3] Z. Ge, D. G. Cahill, and P. V. Braun, "Thermal conductance of hydrophilic and hydrophobic interfaces," Phys. Rev. Lett. 96, 186101 (2006).

[4] S. A. Putnam, D. G. Cahill, P. V. Braun, Z. Ge, and R. G. Shimmin, "Thermal conductivity of nanoparticles suspensions," J. Appl. Phys. 99, 084308 (2006).

[5] O. M. Wilson, X. Hu, D. G. Cahill, and P. V. Braun, "Colloidal metal particles as probes of nanoscale thermal transport in fluids," Phys. Rev. B 66, 224301 (2002).

[6] Z. Ge, Y. Kang, T. A. Taton, P. V. Braun, and D. G. Cahill, "Thermal transport in Au-core polymer-shell nanoparticles," Nano Letters 5, 531-535 (2005).

[7] P. Keblinski, J. A. Eastman, and D. G. Cahill, "Nanofluids for thermal transport," Materials Today 8, 36-44 (2005).

[8] P. Keblinski and D. G. Cahill, "Comment on "Model for heat conduction in nanofluids"," Physical Review Letters 95, 209401 (2005).

[9] S. Shenogin, L. Xue, R. Ozisik, P. Keblinski, and D. G. Cahill, "Role of thermal boundary resistance on the heat flow in carbon-nanotube composites," Journal of Applied Physics 95, 8136-8144 (2004). 
[10] S. T. Huxtable, D. G. Cahill, S. Shenogin, and P. Keblinski, "Relaxation of vibrational energy in fullerene suspensions," Chemical Physics Letters 407, 129-134 (2005).

[11] S. A. Putnam and D. G. Cahill, "Micron-scale apparatus for measurements of thermodiffusion in liquids," Review of Scientific Instruments 75, 2368-2372 (2004).

[12] S. T. Huxtable, D. G. Cahill, and L. M. Phinney, "Thermal contact conductance of adhered microcantilevers," Journal of Applied Physics 95, 2102-2108 (2004).

[13] S. Huxtable, D. G. Cahill, V. Fauconnier, J. O. White, and J.-C. Zhao, "Thermal conductivity imaging at micrometre-scale resolution for combinatorial studies of materials," Nature Materials 3, 298-301 (2004).

[14] H.-K. Lyeo and D. G. Cahill, "Thermal conductance of interfaces between highly dissimilar materials," Phys. Rev. B 73, 144301 (2006).

\section{Invited Presentations}

1. D. G. Cahill, panel discussion on "Nanoscale transport phenomena," ASME Congress 2001, New York, NY, Nov. 11-16.

2. D. G. Cahill, "Thermal transport across solid-solid and solid-liquid interfaces," Mechanical Engineering Department, Purdue University, Nov. 15, 2002.

3. D. G. Cahill, "Time-domain thermoreflectance studies of thin film thermal conductivity and interface thermal conductance, " Seagate Technology, Bloomington, MN, Jan. 30, 2003.

4. D. G. Cahill, "Nanostructured materials and the thermal conductance of interfaces," 27th International Thermal Conductivity Conference, Knoxville, TN, Oct. 26-29, 2003.

5. D. G. Cahill, "Nanostructured materials and the thermal conductance of interfaces," Thermal Management for Micro and Meso Power Systems Conference, Chicago, IL, May 17-18, 2004.

6. D. G. Cahill, "Thermal transport at the nanoscale," Mechanical Engineering Dept. seminar, U. Virginia, Oct. 21, 2004.

7. D. G. Cahill "Thermal transport in nanostructured materials," AVS 51st International Symposium, Anaheim, CA, Nov. 14-19, 2004.

8. D. G. Cahill, "Thermal transport at the nanoscale," Intel Corp., Portland, OR, Dec. 2,2004 .

9. D. G. Cahill, "Thermal transport at the nanoscale," Materials Research Institute seminar, U. Oregon, Eugene, OR, Dec. 3, 2004.

10. D. G. Cahill, "Thermal transport at the nanoscale, IBM Watson Research Center, Yorktown Heights, NY, Feb. 15, 2005. 
11. D. G. Cahill, "Thermal conductance of interfaces," MRS Spring Meeting, San Francisco, CA, March 28-April 1, 2005.

12. D. G. Cahill "Thermal conductance of solid-solid and solid-liquid interfaces," NCN NEMS Workship, Purdue U., April 8, 2005.

13. D. G. Cahill, "Thermal conductance of solid-solid and solid-liquid interfaces," Argonne National Lab, Argonne, IL, Aug. 17, 2005.

14. D. G. Cahill, "Thermal conductance of solid-solid and solid-liquid interfaces," Micro Thermal System Research Center, Seoul National University, Nov. 7, 2005.

15. D. G. Cahill, "Thermal conductance of solid-solid and solid-liquid interfaces," Mech. Eng. Dept., U. Tokyo, Nov. 9, 2005.

16. D. G. Cahill, "Thermal conductance of solid-solid and solid-liquid interfaces," plenary speaker, 26th Japan Thermophysical Properties Conference, Tsukuba, Japan, Nov. 9-11, 2005.

17. D. G. Cahill, "Thermal conductance of interfaces," SRC/NSF 3rd Workshop on Silicon Nanoelectronics and Beyond, Arlington, VA, Dec. 8-9, 2005.

18. P. V. Braun, "Integrated Platforms for Coupling Self-Assembly and Complexity," CINT Workshop, Albuquerque, NM, January 2005.

19. P. V. Braun, Z. Ge, R. G. Shimmin and D. G. Cahill, "Thermal and Photophysical Properties of Functionalized Nanoparticles and Nanoparticle Assemblies," Materials Research Society Fall Meeting, Boston, MA, December 2005.

\section{Awards and Honors, 2002-2006}

\section{D.1 David G. Cahill}

- Fellow of the American Physical Society, 2005; citation: For original and influential contributions to the physics of heat conduction in materials and the evolution of surface morphology during crystal growth and etching.

- Donald Biggar Willett Professor of Engineering, U. Illinois, College of Engineering, 2005-2010.

- Plenary speaker, "Thermal conductance of solid-solid and solid-liquid interfaces," 26th Japan Symposium on Thermophysical Properties, Tsukuba, Nov. 9-11, 2005.

- Willet Faculty Scholar Award, U. Illinois, College of Engineering, 2002-2004. 


\section{D.2 Paul V. Braun}

- Xerox Award for Faculty Research, U. Illinois, College of Engineering, UIUC, 2004.

- Willett Faculty Scholar Award, U. Illinois, College of Engineering, 2002-2008.

- The Robert Lansing Hardy Award of the TMS. Annually awarded to one young materials scientist whose career shows exceptional promise, 2002. 